Ann. Biol. anim. Bioch. Biophys., I970, 10 (2), 203-208.

\title{
OBSERVATIONS SUR L'INFLUENCE IE LA PHOTOPÉRIODE SUR L'ACTIVITÉ SEXUELLE CHEZ MICROCEBUS MURINUS (MILLER, 1777) EN CAPTIVITÉ
}

\author{
Arlette PETTER-ROUSSEAUX \\ Laboratoive d'Écologie, \\ Muséum d'Histoire naturelle, 91 - Brunoy
}

RF́:SUMÉ

L'activité sexuelle de Microcebus murinus (Miller, I777), petit Lémurien nocturne de Madagascar, est sensible à la stimulation photopériodique. La saison de reproduction - d'octobre à février à Madagascar - est inversée par le changement d'hémisphère. Les observations faites en captivité à Paris, en éclairement naturel, pendant 6 années, ont montré que pour la majorité des femelles (64 p. Ioo), le premier œstrus de la saison a lieu en avril, et surtout dans la deuxième quinzaine d'avril.

Un éclairement artificiel de durée constante I 6 L-8 D, à contre-saison, a provoqué l'apparition des cestrus au bout de trois mois.

La rapidité d'adaptation au changement d'hémisphère est mise en évidence : des femelles, importées fin décembre, pendant la période d'activité à Madagascar, entrent en repos, puis reprennent leur activité au printemps suivant, 4 ou 5 mois plus tard. Des femelles importées le I er février ne répondent pas à la stimulation et ne montrent pas d'œestrus dans l'année : une période réfractaire est ainsi suggérée.

Les études sur le terrain, de plus en plus nombreuses, montrent que la plupart des Primates ont, à l'état naturel, une saison d'activité sexuelle privilégiée, dont le résultat est de grouper les naissances à la saison qui semble la plus favorable.

On recherche habituellement dans l'environnement (rythme saisonnier d'éclairement, conditions climatiques diverses, nourriture) le facteur qui déclenche l'activité sexuelle. Les facteurs éclairement et température ne sont en général pas pris en considération, les Primates se trouvant à des latitudes où les différences saisonnières restent très faibles. Par contre, l'alternance des pluies et de la sécheresse qui modifie 
la végétation et par suite 1'alimentation en général, a été considérée par la plupart des auteurs comme influençant l'activité sexuelle. (STTRUHSAKER, I967, sur Cercopithecus aethiops à Amboseli; GAUTHIER, I968, sur Myopithecus talapoin au Gabon ; VANDENBergh et Vessex, I968, sur Macacus rhesus à Cayo Santiago). Cependant, la concordance entre période d'accouplement ou de mise bas d'une part, et saison des pluies d'autre part, et les comparaisons entre espèces voisines ou animaux de la même espèce vivant sous des climats légèrement différents sont délicates à établir. GARTLAN (1969) remarque que Cercopithecus aethiops dans deux régions situées à 300 miles l'une de l'autre et ayant un régime de pluies similaire, se reproduit en deux saisons opposées (réserve d'Amboseli et île de Lolui, lac Victoria).

Les études de MASAO KaWAI (I967) montrent la difficulté de rattacher à un facteur principal le déclenchement de la saison d'activité sexuelle chez 25 troupes de Macaca fuscata qui s'accouplent, au Japon, au cours de 3 ou 4 mois entre septembre et avril.

Plusieurs auteurs ont signalé un décalage progressif de la saison d'accouplements chez des Primates transportés d'un hémisphère à l'autre. (KOFORD, I965; DU MOND, I967).

En captivité, nourris de façon régulière toute l'année, les Primates supérieurs ont une saison d'accouplements très étendue, parfois à toute l'année, sans période privilégiée, (VAN WAGENEN, I967, sur Macaca mulatta), ou avec la persistance d'un pic (VICE, I968, sur Papio papio et Papio cynocephalus).

Chez les Lémuriens, le processus varie suivant les espèces. L'activité sexuelle est saissonnière chez Perodicticus potto au Gabon (CharLes-Dominique, I968) mais, en captivité, cet animal montre des cycles réguliers toute l'année (MANLEY, I965; IoAnNou, I966). Chez Loris tardigradus, il y aurait deux saisons d'œestrus dans la nature (RAMASWAMI, I962) aussi bien qu'en captivité (MANIEY, I965); mais la spermatogenèse se ferait toute l'année (RAmakrishna, r967).

Les Lémuriens malgaches, à Madagascar, ont également une activité sexuelle saisonnière, variant suivant les espèces : de septembre à janvier chez les Cheirogaleinae, de mai à juillet chez les Lepilemurs, d'avril à juin chez les Lemurs, en janvierfévrier chez les Propithèques (PETTER-RoussEAux, I968). Ces animaux sont caractérisés par le fait qu'ils inversent leur période d'activité sexuelle en changeant d'hémisphère, et que, même en captivité dans l'hémisphère nord, cette activité reste saisonnière, et de même ordre de durée qu'à Madagascar. Ceci se produit aussi bien chez les petites espèces nocturnes, comme nous l'avons constaté nous-même (PETTERROUSSEAUX et Bourlitere, I959) que chez les grandes espèces diurnes élevées en jardin zoologique. Nous avons cherché à préciser le rôle de la photopériode sur l'espèce Microcebus murinus (MILLER I777) que nous élevons depuis une quinzaine d'années : en particulier :

I. Dans quelle mesure des animaux importés, à la fin de leur période d'activité sexuelle à Madagascar, pouvaient entrer de nouveau en activité quelques mois plus tard.

2. L,e temps nécessaire à déclencher l'activité sexuelle chez des animaux au repos, en les soumettant à un éclairement artificiel. Nous avons choisi pour cela un régime de jours longs I6 L- $8 \mathrm{D}$, les animaux ayant été au préalable exposés à un régime $8 \mathrm{~L}-\mathrm{I} 6 \mathrm{D}$ pendant un mois. 


\section{MATÉRIEI, E'T MÉTHODES}

La difficulté d'obtenir ces animaux, qui sont rares — ne vivant qu'à Madagascar -- et protégés, nous a obligé à travailler sur un petit nombre d'individus. L'importance de notre élevage a varié d'une quinzaine à une trentaine d'individus. L'effectif était trop réduit notamment pour être réparti en plusieurs lots soumis à des expériences simultanées. l'ensemble des animaux était donc soumis chaque année à des conditions définies, et nous avons tiré nos conclusions de la comparaison des années successives.

Les Microcèbes (animal adulte : $60 \mathrm{gr}$ en moyenne) sont élevés à une température voisine de $25^{\circ} \mathrm{C}$; les cages sont cubiques, de $60 \mathrm{~cm}$ de côté, garnies de copeaux, et contiennent $\mathrm{I}$ à 4 individus. Elles sont munies d'un ou deux nichoirs en bois pourvus d'une petite ouverture arrondie, de 4 à $5 \mathrm{~cm}$ de diamètre. La nourriture se compose de fruits variés, lait additionné de vitamines, miel, pain d'épices, vers de farine, viande crue.

Ces animaux sont nocturnes et ne sortent de leurs niches qu'à la nuit tombante. Pendant la journée, à l'intérieur des nichoirs, ils reçoivent une quantité de lumière extrêmement faible.

A l'extérieur des nichoirs, la quantité de lumière reçue varie de 50 à i 50 lux suivant l'emplacement, dans les pièces éclairées artificiellement par des tubes "Iumière du jour" de $40 \mathrm{~W}$.

L'œstrus est reconnaissable extérieurement au gonflement ct à l'ouverture de la vulve qui se referme progressivement quelques jours plus tard. Les $q$ ont toujours été au voisinage de cages contenant un $\sigma^{*}$.

\section{RÉSULTATS}

\section{A. - Adaptation au changement d'hémisphère}

La rapidité de l'adaptation au changement d'hémisphère a été mise en évidence dans les deux expériences suivantes :

I. 9 femelles importées le 2 I décembre, donc en période d'activité sexuelle à Madagascar, entrent en repos ; les œstrus réapparaissent à partir de fin avril, c'està-dire environ 4 ou 5 mois seulement après leur saison d'activité précédente.

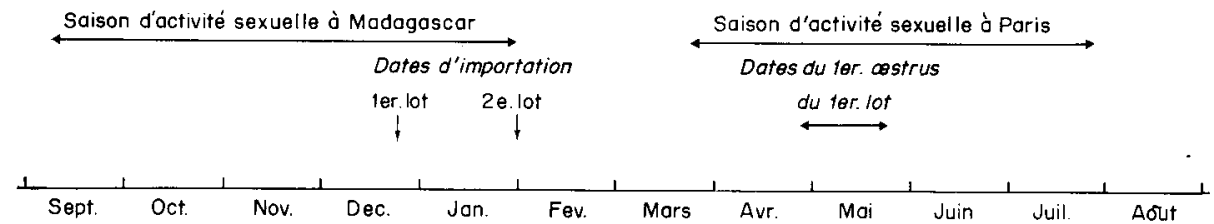

FIG. I. - Adaptation au changement d'hémisphève : le deuxième lot, importé fin janvier, n'entre pas en oestrus.

2. I3 femelles (Io adultes et 3 nées au début de janvier) entrent en France le I $^{\text {er }}$ février, après leur période de reproduction à Madagascar. Une seulement d'entre elles (jeune) entre en œstrus cette année-là (9 juillet).

Ces femelles n'étaient ni gestantes ni allaitantes à leur arrivée. Elles avaient été collectées au cours des 2 ou 3 mois précédents et pouvaient avoir eu un jeune lors de la saison de reproduction malgache en novembre et décembre.

\section{B. - Moment d'apparition de l'cestrus chez des animaux élevés en France}

En éclairement naturel, à Paris, nous avons relevé les premières dates d'œestrus dans la deuxième quinzaine de mars; les chiffres que nous utilisons dans le graphique suivant, correspondent à 6 années d'observations, sur un total de 97 ostrus. La 
majorité des cestrus a lieu en avril (64 p. Ioo) et particulièrement dans la $2^{\mathrm{e}}$ quinzaine (44 p. Ioo) ; les derniers ont lieu dans la seconde quinzaine de juin. Les ostrus tardifs sont d'ailleurs souvent le fait de femelles très âgées ou très jeunes, ou en mauvaises conditions physiologiques.

\section{C. - Animaux placés en jours longs à contre-saison}

Chez les animaux soumis à un éclairement artificiel I6 L-8 D, à partir du 20 septembre, nous avons assisté à l'apparition des premiers œestrus dans la seconde quinzaine de décembre ; les chiffres donnés correspondent à I 7 œstrus. La plupart d'entre eux (64 p. Ioo) a lieu en janvier et pendant la première quinzaine (4I p. I00).

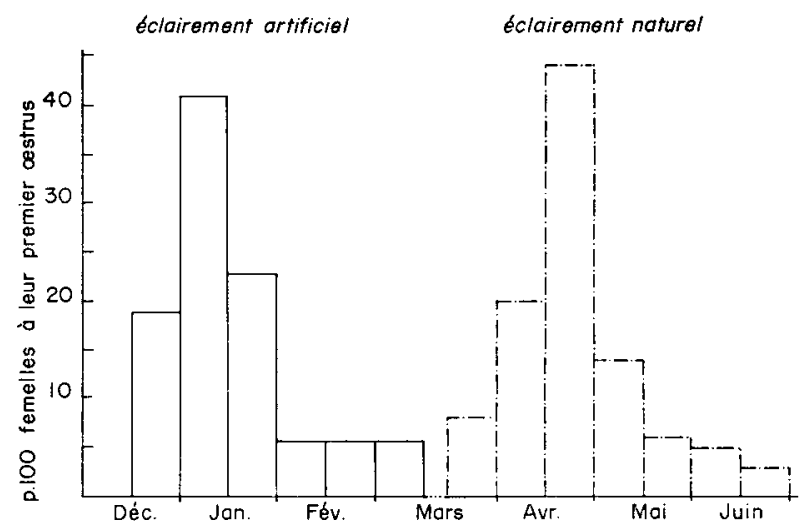

FIG. 2. --- Pourcentage de femelles à leur premier astrus chaque quinzaine de chaque mois, sous un éclairement naturel a Paris (courbe -.-.-) et dans l'expérience $\mathrm{I} 6 \mathrm{~L}-8 \mathrm{D}$, commencée le 20 septembre (-- - ). Toutes les femelles entrent en cestrus.

\section{DISCUSSION}

Les cestrus sont très groupés, 64 p. Ioo d'entre eux ayant lieu, en éclairement naturel de Paris, pendant le mois d'avril; dans l'expérience d'éclairement artificiel cette même proportion se retrouve pour les œstrus ayant lieu en janvier ; nous avons représenté sur un graphique (fig. 2) 1e nombre des œstrus en fonction du temps en éclairement naturel et au cours de l'expérience. La ressemblance entre les deux courbes est remarquable. Dans le premier cas, la majorité des cestrus a lieu 3 mois et demi après le début de l'accroissement naturel de longueur des jours; dans le second cas, on retrouve cette majorité 3 mois et demi après le début du régime d'éclairement artificiel I6 L-8 D. Ceci laisserait supposer que cette durée de 3,5 mois est nécessaire à la stimulation; dans cette expérience elle semble indépendante du régime d'accroissement des jours; de plus, un accroissement progressif ne paraît pas indispensable.

Dans la première expérience d'importation, des femelles importées pendant leur période d'activité, entrent immédiatement en repos (passant aux jours courts de l'hiver de Paris), puis entrent de nouveau en activité au printemps, 4 mois seulement après (fig. I). 
Les jours du début de janvier, bien que déjà croissants, sont courts par rapport aux jours malgaches de décembre ( 13 h 30 environ), ils n'ont pas stimulé ces femelles qui ont ainsi bénéficié d'une période de repos de 3 semaines au moins.

Dans la seconde expérience, l'importation a lieu à la fin de la saison d'activité malgache, un mois plus tard, au I er février. A cette époque les jours croissent très rapidement et les animaux sont soumis à une nouvelle stimulation lumineuse, la durée des jours rejoignant alors très vite celle à laquelle ils étaient soumis à Madagascar.

Il est possible que cette nouvelle stimulation ait eu lieu pendant une période réfractaire qui suivrait la saison d'activité. Mais rien ne permet encore de préciser ce point.

\title{
CONCLUSIONS
}

L'activité sexuelle du. Microcebus murinus dépend de la photopériode.

La stimulation photopériodique se manifeste après 3 mois au minimum et en moyenne après 3,5 mois ; il n'est pas nécessaire que les jours s'accroissent progressivement.

Des femelles importées en France pendant leur saison d'activité sexuelle à Madagascar, sont susceptibles d'entrer de nouveau en activité 4 ou 5 mois plus tard, sous l'influence des jours croissants.

On peut supposer l'existence d'une période réfractaire à la fin de la saison d'activité sexuelle.

Rę̧u pour publication en février 1970.

\section{SUMMARY}

\author{
EFFECT OF PHOTOPERIOD ON SEXUAL BEHAVIOUR \\ IN THE CAPTIVE MICROCEBUS MURINUS LEMUR.
}

The breeding activity of Microcebus murinus (a small night Madagascan Lemur) is photoperiodically stimulated. The breeding season extending from October to February in Madagascar is changed over in France. Observations for 6 years under natural conditions of light in Paris show that 64 per roo females experienced their first ostrus in April (mainly during the 2 nd fortnight).

I 6 light/8 dark hour artificial lighting irrespective of the season induced the onset of cestrus within a 3.5 month lapse of time.

The female Microcebus murinus shows great adaptive abilities : females brought from Madagascar at the end of December, i. e. during the breeding season, came into quiescence for 4 to 5 months until Spring. Females brought on the Ist of February, i. e. terminating their breeding season, exhibited no reproductive response to light changes, which suggests the existence of a refractory period.

\section{RÉFÉRENCES BIBLIOGRAPHIQUES}

Charles-Dominigue P., I968. Reproduction des Lorisidés africains. in R. Canivenc. Cycles gémitaux saisonniers des Mammifères sauvages, Entretiens de Chizé, Masson Paris, z-9.

Du Mond F. V., Hutchinson T. C., r967. Squirrel-monkey Reproduction : The " Fatted " male phenomenon and seasonal spermatogenesis. Science, 158, ro67-1070. 
livans C. S., Goy R. W., 1968. Social behaviour and reproductive cycles in captive ring-tailed lemurs (Lemur catta). J. Zool., 156, I8I-197.

Gartlan J. S., rg69. Sexual and maternal behaviour of the vervet monkey (Cercopithecus aethiops) J. Reprod. Fert., Suppl., 6, 137 -I50.

Gauthier-hion A., Ig68. Étude du cycle annuel de reproduction du Talapoin (Myopithecus talapoin) vivant dans son milieu naturel. Biol. Gabon., 2, I63-173.

Ioannou J. M., I966. The costrus cycle of the Potto. J. Reprod. Fert., 11, 455-457.

Jolly A., I966. Lemur behavior. The University of Chicago Press.

Koford C. B , 1965. Population dynamics of rhesus monkeys on Cayo-Santiago, in De Vore Primate behavior Holt, Rinehart and Winston.

Mac Roberts M. M., Mac Robrrts I3., I966. The animal reproductive cycle of the barbary ape (Macaca sylvana) in Gibraltar. Amer. J. Phys. Anthropol., 25, 299-304.

Manley G. H., I965. Reproduction in Lorisoid primates. J. Reprod. Fevt., 9, 390-39I.

Petter-Rousseaux A., Bourlierf F., 1959. La périodicité sexuelle des femelles d'un Primate inférieur, Je Microcebus murimus. Effet d'une inversion saisonnière de l'éclairement. $\quad C$. R. Soc. Biol., $153,226$.

Petter-Rousslaux A., I968. Cycles génitaux saisonniers des Lémuriens malgaches, in R. Canivenc Cycles génitaux saisonniers de Mammifères sauvages. Entretiens de Chizé, Masson, Paris, I I-22.

Pickining D. Ji., r 968 . Reproduction characteristics in acolony of laboratory confined mulat ta macaque monkeys. Folia Primatol., 8, I69-I79.

Ramakrishna P. A., Prasad M. R. N., Ig62. Reproduction in the male slender loris, Curr. Sci. India, 31, 468-469.

Ramaswami L. S., Anand Klyar T. C., I962. Reproductive cycle of the slender loris. Vaturwiss, 5, II5-II6.

Struhsaker T. T., I967. Behavior of vervet monkeys (C, aethiops). Univ. California Public. Zool, 82, 74 p.

VANDENBERGH J. G., VeSsey S., r 968 . Seasonal breeding of free-ranging rhesus monkeys and related ecological factors. J. Reprod. Fert., 15, 7I-79.

Vice T. E., Britton M. A., Ratner I. A., Kalert S. S., i968. Care and raising of newborn baboons. J. small animal Pract., 9, I24-129.

VAN Wagenen G., I967. Fertility of the colony-born male macaque. Folia primatol., 5, 24 I-246. 